\title{
ERRATUM FOR "NON SINGULAR HAMILTONIAN SYSTEMS AND GEODESIC FLOWS ON SURFACES WITH NEGATIVE CURVATURE"
}

\author{
E. A. Lacomba and J. G. Reyes
}

A mistake was found in the reasoning leading to a Lagrangian which we considered as equivalent from the formula for the action $S(\gamma)$ below the classical mechanical problem (3) on [1, page 271]. It turns out that the new Lagrangian, corresponding to the conformal metric $g_{h}$ at the bottom of the page, is equivalent to the classical mechanical Lagrangian, but only for the case of one degree of freedom. This means that Proposition 2 is not valid for two or more degrees of freedom. However, the resulting geodesic flow has many qualitative features similar to those of the original mechanical problem. Indeed, computing the Euler-Lagrange equations for

$$
L=\frac{1}{\sqrt{2}} \frac{\delta_{i j} \dot{x}^{i} \dot{x}^{j}}{\sqrt{h-U}}
$$

subject to the constraint $\frac{1}{2} \delta_{i j} \dot{x}^{i} \dot{x}^{j}-U(x)=h$, we find, after a long computation, the system of differential equations

$$
2(h-U)[\ddot{\mathbf{x}}+\nabla U]=(\dot{\mathbf{x}} \cdot \dot{\mathbf{x}}) \nabla U-\dot{\mathbf{x}}(\nabla U \cdot \dot{\mathbf{x}}) .
$$

Since the right hand side is orthogonal to the velocity $\dot{\mathbf{x}}$, we verify that indeed the energy is a first integral. For $\mathrm{n}=1$ degree of freedom, the above equation becomes $2(h-U)\left[\ddot{x}+U^{\prime}\right]=\dot{x}^{2} U^{\prime}-\dot{x} U^{\prime} \dot{x}=0$ or $\ddot{x}+U^{\prime}=0$, the same than for the original classical mechanical Lagrangian. But if $n \neq 1$ the right hand side is not zero in general, as can be seen with the example $n=2$ and $U=\frac{1}{2}\left[\left(x^{1}\right)^{2}+\left(x^{2}\right)^{2}\right]$. Then the two components of the right hand side of the equations of motion become $\dot{x}^{2}\left(x^{1} \dot{x}^{2}-x^{2} \dot{x}^{1}\right)$ and $-\dot{x}^{1}\left(x^{1} \dot{x}^{2}-x^{2} \dot{x}^{1}\right)$ respectively; these expressions can be zero only if the velocity is zero or if it is orthogonal to the position vector.

For now, we are left with results about either one of the two systems, as follows. The remaining part of Section 2 is valid for the geodesic conformal system $\left(\operatorname{int}\left(M_{h}\right), g_{h}, U\right)$. These are more precisely

Theorem 2, classifying the sign of Gaussian curvature in terms of the gradient and the Laplacian of the potential, as well as Corollary 1. 
Lemma 1, stating that geodesics escaping to infinity have infinite length.

Lemma 2, stating that any geodesic going to the curve $\Gamma=\{U(x, y)=$ $0\}$ has finite length. Also Corollary 2, stating that the geodesic system is not geodesically complete.

All the results in Section 3 are stated for the original classical mechanical problem. Hence, they are all true, except for the comment about examples in Section 5 on the final line of Section 3.

Section 4 refers to the geodesic conformal system $\left(\operatorname{int}\left(M_{h}\right), g_{h}, U\right)$, but not all the results remain valid since it was partly based on Section 3. We do not know if the following results still hold:

Theorem 6. Given any direction $z=\arctan (\alpha)$ in the Hill's region and any point $P \in \operatorname{int}\left(M_{h}\right)$, there exists a unique geodesic $\gamma_{P}$ through $P$ having positively asymptotic direction $z$. Analogously for the negatively asymptotic case.

Corollary 5. If $P \in \operatorname{int}\left(M_{h}\right)$ and $z \in M_{h}(\infty)$, then there exists a unique geodesic $\gamma \subset M_{h}$ defined for any big enough positive time, such that $\gamma(0)=P$ and $\gamma(+\infty)=z^{+}$. Similarly for the case $\gamma(0)=P$ and $\gamma(-\infty)=z^{-}$.

Theorem 7. Given two points $z_{1}, z_{2}$ in $\overline{M_{h}}(\infty)$, there exists a unique geodesic $\gamma(t)$ such that $\gamma \subset z_{1}^{-}$and $\gamma \subset z_{2}^{+}$.

Corollary 7. Any geodesic $\gamma \subset \overline{M_{h}}$ can be written in the form $\gamma=$ $z_{1}^{-} \cap z_{2}^{+}$as the intersection of two classes of geodesics.

The last three theorems in the paper also remain open, since they relate the original mechanical system with the geodesic flow. They are:

Theorem 9. The set of physical curves in the configuration space $M_{h}$ is related, as a one-dimensional foliation, to the geodesic flow of a space homeomorphic to the unit disk, associated to a conformal quasi-complete metric with negative curvature.

Theorem 10 (Geometry of the Repulsive Isosceles Problem). If the masses satisfy the relation $\mu \geq 2 m$, we have that the set of physical curves of this problem is related, as a one-dimensional foliation, to the geodesic flow in a space homeomorphic to the unit disk, with respect to a conformally Euclidean quasi-complete metric with negative curvature. 
Theorem 11 (Geometry of the Repulsive Rhomboidal Problem). If the masses satisfy the relation $\frac{m_{1}}{2} \leq m_{3} \leq 2 m_{1}$, we have that for a fixed energy level $h>0$, the set of physical curves is related, as a onedimensional foliation, to the geodesic flow in a space homeomorphic to the unit disk with respect to a quasi-complete conformal metric with negative curvature.

We believe that all the other results in Section 4 remain valid for the geodesic system.

\section{Reference}

[1] E. A. Lacomba And J. G. Reyes, Non singular Hamiltonian systems and geodesic flows on surfaces with negative curvature, Publ. Mat. 42 (1998), 267-299.

Departamento de Matemáticas

Universidad Autónoma Metropolitana-Iztapalapa

Apartado postal 55-534

09340 México, D.F. México

E-mail address: lace@xanum.unam.mx

E-mail address: revg@xanum.unam.mx

Rebut el 20 de setembre de 1999. 